

open research

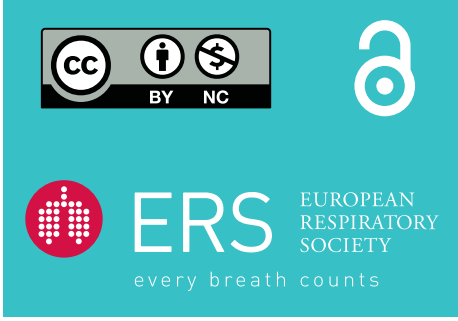

\section{Pneumatocoeles after bronchoscopic lung volume reduction with valves}

\section{To the Editor:}

Based on the positive results of five randomised controlled trials, bronchoscopic lung volume reduction (BLVR) using Zephyr endobronchial valves (EBV) implantation has been approved for the treatment of patients with severe emphysema and little to no collateral ventilation [1]. These one-way valves produce an atelectasis (or volume reduction) of the target lobe, which leads to decreased hyperinflation and ultimately improvement in exercise capacity. However, EBV therapy is associated with a number of potential adverse events, pneumothorax being the most frequent and threatening complication, occurring in $\sim 20 \%$ of cases [1], and whose treatment is based on chest tube insertion, suction and in cases of prolonged air leaks, valve(s) removal [2]. We report a very rare complication of EBV-induced pneumothorax treatment: a pneumatocoele. We propose a mechanistic explanation and preventive measures.

A 64-year-old male was referred to our institution for BLVR. The patient was a former smoker (30 pack-years) with no other medical history, suffering from severe dyspnoea on exertion (mMRC 4) despite optimal medical treatment including tiotropium and salmeterol, and formal rehabilitation programmes. Pulmonary function tests showed a $2.50 \mathrm{~L}$ forced vital capacity (FVC $64 \%$ pred), $0.90 \mathrm{~L}$ forced expiratory volume in $1 \mathrm{~s}\left(\mathrm{FEV}_{1} ; 29 \%\right.$ pred), $0.3 \mathrm{FEV}_{1} / \mathrm{FVC}$ ratio and $5.3 \mathrm{~L}$ residual volume (RV; $219 \%$ pred). At exercise, the patient had a significant dynamic hyperinflation with a drop in inspiratory capacity (IC) from $1.87 \mathrm{~L}$ to $1.15 \mathrm{~L}$. Chest computed tomography (CT) scan revealed heterogeneous severe emphysema with predominant destruction of the upper lobes. Right upper lobe was found to be the best target via quantitative tomoscintigraphy (4\% perfusion). The StratX, (PulmonX, Redwood City, CA, USA) software analysis confirmed the target $(79 \%$ and $60 \%$ of parenchyma with less than $-910 \mathrm{HU}$ and -950 HU densities, respectively; no collateral ventilation). Four Zephyr endobronchial valves (PulmonX) were placed in the right upper lobe through a flexible bronchoscope under general anaesthesia. A chest radiograph was rapidly performed in the post-operative recovery room due to acute dyspnoea, revealing a complete pneumothorax. A chest tube was inserted and put to suction $\left(-20 \mathrm{~cm} \mathrm{H}_{2} \mathrm{O}\right)$, the patient describing a violent and unusual chest pain immediately after suction. Air leak (bubbling) stopped after less than $24 \mathrm{~h}$, and the drain was removed after 24 additional hours without suction (i.e. $48 \mathrm{~h}$ after its insertion). The day after, a CT scan was performed due to moderate haemoptysis $\left(50 \mathrm{~mL} \cdot \mathrm{day}^{-1}\right)$ and air-fluid levels on the radiograph. It revealed two intra-parenchymal (right middle lobe and right upper lobe) air cavities with hydric levels, corresponding to pneumatocoeles partially filled with liquid (likely blood) (figure 1b). During the next few days, mild haemoptysis persisted but the patient's condition improved, allowing for oxygen weaning. Another CT scan was performed 8 days after the procedure, showing stability in size of the pneumatocoeles, with a greater fluid content, and a complete atelectasis of the right upper lobe (figure 1c). We decided not to remove valves and the patient was discharged on antibiotics. During the following weeks, the patient experienced a significant improvement in his dyspnoea, which dropped to mMRC 1. A 1-month CT scan showed a complete atelectasis of the target upper right lobe and a favourable evolution of the cavities, both decreasing in size (figure 1d), and 5 months after the procedure, both pneumatocoeles were fully healed (figure 1e). Pulmonary function tests

@ERSpublications

Pneumatocoele is a very rare complication of Zephyr EBV, probably due to chest tube insertion and suction of a trapped and emphysematous lung. Complete healing and functional improvements are possible without the need for valve removal. https://bit.ly/2K84Vjl

Cite this article as: Lestelle F, Fumat R, Didier A, et al. Pneumatocoeles after bronchoscopic lung volume reduction with valves. ERJ Open Res 2021; 7: 00747-2020 [https://doi.org/10.1183/ 23120541.00747-2020].

Copyright $\odot$ The authors 2021. This version is distributed under the terms of the Creative Commons Attribution NonCommercial Licence 4.0. For commercial reproduction rights and permissions contact permissions@ersnet.org 


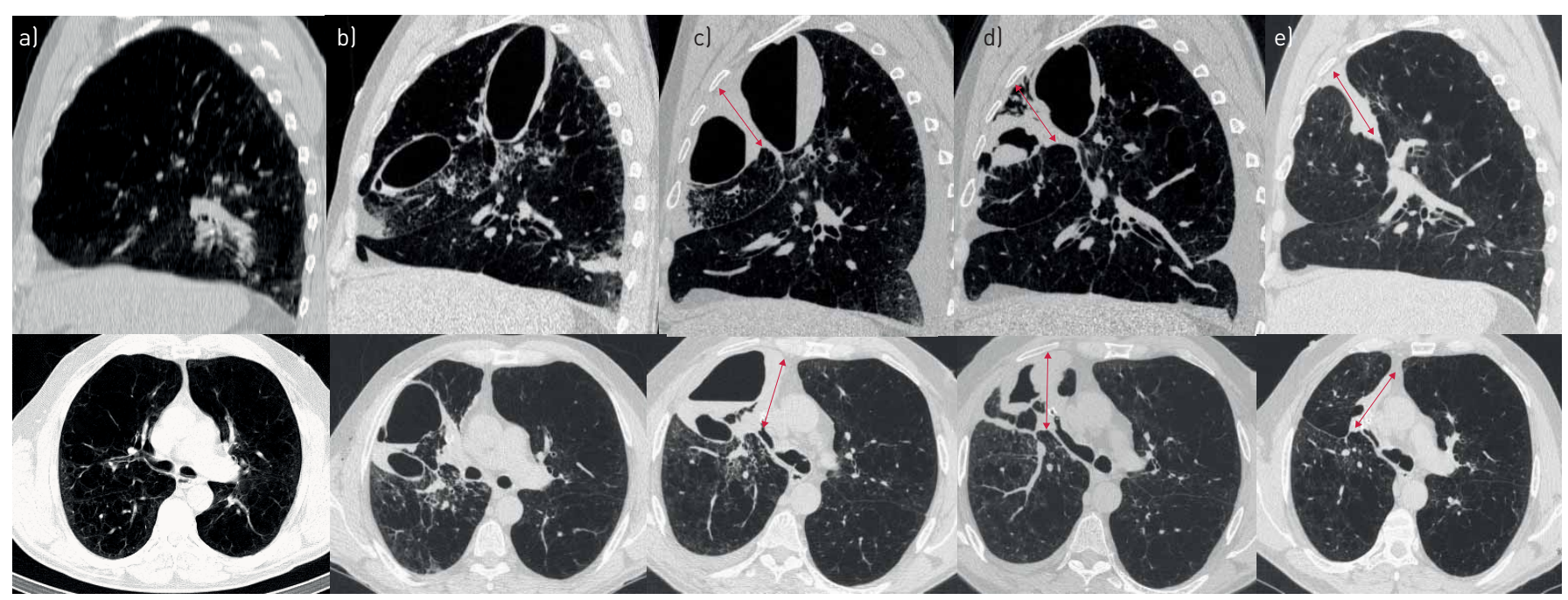

FIGURE 1 a) Pretreatment computed tomography (CT) scan. b) CT scan 4 days after endobronchial valve (EBV) treatment showing pneumatocoeles in the right middle lobe and right upper lobe. The expected right upper lobe atelectasis was observed at day 8 (c) (red arrows), a decrease in size of the cavities after 3 weeks (d) and a complete healing after 5 months (e).

at 5 months demonstrated great improvement: FVC, $3.7 \mathrm{~L}$ (95\% pred, $48 \%$ improvement); $\mathrm{FEV}_{1}, 1.6 \mathrm{~L}$ (52\% pred, $75 \%$ improvement); total lung capacity, $7.8 \mathrm{~L}$ (117\% pred); and RV $4 \mathrm{~L}$ (167\% pred, $25 \%$ decrease). Furthermore, no more dynamic hyperinflation was noted, with a $2.5 \mathrm{~L}$ IC before exercise, remaining stable during it. The 6-min walk distance was improved by $150 \mathrm{~m}$ (270 to $420 \mathrm{~m}$ ).

BLVR with one-way EBVs has been demonstrated to improve pulmonary function, exercise capacity and quality of life in patients with both heterogeneous and homogeneous emphysema without collateral ventilation [3]. The most common complications of EBV placement include pneumothorax, pneumonia, respiratory exacerbations and valve migrations [4].

To the best of our knowledge, this is the second reported case of pneumatocoele after EBV therapy [5]. Pneumatocoeles are commonly seen after blunt pulmonary trauma, their exact pathogenesis remaining uncertain [6]. They correspond to thin-walled, gas-filled spaces in the lung [7]. In our case, the pathogenesis could be explained by a fragility of the emphysematous lung parenchyma, which was torn after the management of the pneumothorax with a chest tube insertion and rapid negative pressure suction. The sole other report of EBV-induced pneumatocoele also occurred after pneumothorax and chest tube insertion [5]. Another potential explanation is a trauma induced by the chest tube itself, even though a direct trauma would likely induce a single pneumatocoele. We suggest that after chest tube insertion for EBV-induced pneumothorax, suction should be soft and delayed to avoid this complication.

The decision of whether or not to remove one or all of the inserted valves usually depends on the persistence of air leak, precluding drain weaning [2]. In this case of pneumatocoele, the chest tube was rapidly removed. However, though the idea that valve(s) removal would relieve atelectasis, limit traction on the parenchyma and thus facilitate healing has been discussed, we decided for close expectant management because the patient was clinically improving. In the other report, three valves were removed, and the patient did not derive any benefit from the treatment. Our report demonstrates EBV-induced pneumatocoele can heal without removal, months after the procedure, with clinical improvement.

The main differential diagnosis of pneumatocoele is the persistent interlobar pneumothorax $[2,8]$, sometimes with trapped interlobar air without air leak, that is difficult to exclude with imaging alone. In this case, the unusually violent chest pain after suction instauration and subsequent abundant and persistent $\left(50 \mathrm{~mL} \cdot \mathrm{day}^{-1}\right)$ haemoptysis could suggest a parenchymal trauma, which may have occurred due to aspiration of a trapped lung.

The clinical outcomes of this patient have been paradoxically remarkable, like for the other reported case [5], with a deep reduction in $\mathrm{RV}$, resulting in a $75 \%$ improvement in $\mathrm{FEV}_{1}$, translating into a dramatic clinical improvement. The benefit might have two distinct mechanisms: the common, expected, EBV-induced atelectasis; and a mechanical effect of the retraction of the parenchyma following pneumatocoele healing.

In conclusion, pneumatocoele should be considered as a potential "complication of a complication (pneumothorax)" after EBV treatment, potentially caused by suction of a trapped emphysematous lung. We suggest that after chest tube insertion for EBV-induced pneumothorax, suction should be delayed to 
avoid this complication. In the absence of prolonged air leak, valve removal should be decided on a case-by-case basis, depending on clinical evolution, but our report suggests that pneumatocoele can evolve favourably while leaving valves in place.

Francois Lestelle ${ }^{1}$, Romane Fumat ${ }^{1}$, Alain Didier ${ }^{1}$, Samia Collot $^{2}$, Thomas Egenod $^{3}$, Guillaume Faviez ${ }^{1}$, Christophe Hermant ${ }^{1}$, Gavin Plat $\oplus^{1}$ and Nicolas Guibert ${ }^{1}$

${ }^{1}$ Pulmonology Dept, Larrey University Hospital, Toulouse, France. ${ }^{2}$ Radiology Dept, Rangueil University Hospital, Toulouse, France. ${ }^{3}$ Pulmonology Dept, Dupuytren University Hospital, Limoges, France.

Correspondence: Nicolas Guibert, Thoracic Endoscopy Unit, Larrey University Hospital, 24 chemin de Pouvourville, Toulouse 31000, France. E-mail: guibert.n@chu-toulouse.fr

Received: 13 Oct 2020 | Accepted: 2 Dec 2020

Patient consent: The patient gave his consent for the publication of his case.

Availability of supporting data: All data are available immediately.

Authors' contributions: F. Lestelle and N. Guibert collected data and drafted the manuscript; S. Collot interpreted the computed tomography images; G. Faviez interpreted the pulmonary function tests; all other authors revised the manuscript and approved the final version.

Conflict of interest: None declared.

\section{References}

1 Labarca G, Uribe JP, Pacheco C, et al. Bronchoscopic lung volume reduction with endobronchial zephyr valves for severe emphysema: a systematic review and meta-analysis. Respiration 2019; 98: 268-278.

2 Valipour A, Slebos D-J, de Oliveira HG, et al. Expert statement: pneumothorax associated with endoscopic valve therapy for emphysema: potential mechanisms, treatment algorithm, and case examples. Respiration 2014; 87: 513-521.

3 Slebos D-J, Shah PL, Herth FJF, et al. Endobronchial valves for endoscopic lung volume reduction: best practice recommendations from expert panel on endoscopic lung volume reduction. Respiration 2017; 93: 138-150.

4 Fiorelli A, D’Andrilli A, Bezzi M, et al. Complications related to endoscopic lung volume reduction for emphysema with endobronchial valves: results of a multicenter study. J Thorac Dis 2018; 10: Suppl. 27, S3315-S3325.

5 Skowasch D, Pizarro C, Valipour A, et al. Endobronchial valve-induced pneumatocele: a case report. Pneumologie 2013; 67: 639-640.

6 Gavelli G, Canini R, Bertaccini P, et al. Traumatic injuries: imaging of thoracic injuries. Eur Radiol 2002; 12: 1273-1294.

7 Hansell DM, Bankier AA, MacMahon H, et al. Fleischner Society: glossary of terms for thoracic imaging. Radiology 2008; 246: 697-722.

8 Mitropoulou G, Casutt A, Lovis A. Long-standing air-leak after valve lung volume reduction necessitating lobectomy. Arch Bronconeumol 2016; 52: 495-497. 